\title{
Reliability assessment of MVP-BURN and JENDL-4.0 related to nuclear transmutation of light platinum group elements
}

\author{
Atsunori Terashima ${ }^{1,2, a}$, Mikael Nilsson ${ }^{2}$, Masaki Ozawa $^{3}$, and Satoshi Chiba ${ }^{3}$ \\ ${ }^{1}$ Department of Nuclear Engineering, Graduate School of Science and Engineering, Tokyo Institute of Technology, 2-12-1-N1-9 \\ Ookayama, Meguro-ku, Tokyo 152-8550, Japan \\ 2 Department of Chemical Engineering and Materials Science, The Henry Samueli School of Engineering, University of California, \\ Irvine, 916 Engineering Tower, Irvine, CA 92697-2575, USA \\ ${ }^{3}$ Laboratory for Advanced Nuclear Energy, Institute for Innovative Research, Tokyo Institute of Technology, 2-12-1-N1-9 Ookayama, \\ Meguro-ku, Tokyo 152-8550, Japan
}

\begin{abstract}
The Aprés ORIENT research program, as a concept of advanced nuclear fuel cycle, was initiated in FY2011 aiming at creating stable, highly-valuable elements by nuclear transmutation from fission products. In order to simulate creation of such elements by $(n, \gamma)$ reaction succeeded by $\beta^{-}$decay in reactors, a continuous-energy Monte Carlo burnup calculation code MVP-BURN was employed. Then, it is one of the most important tasks to confirm the reliability of MVP-BURN code and evaluated neutron cross section library. In this study, both an experiment of neutron activation analysis in TRIGA Mark I reactor at University of California, Irvine and the corresponding burnup calculation using MVP-BURN code were performed for validation of the simulation on transmutation of light platinum group elements. Especially, some neutron capture reactions such as ${ }^{102} \mathrm{Ru}(n, \gamma){ }^{103} \mathrm{Ru},{ }^{104} \mathrm{Ru}(n, \gamma){ }^{105} \mathrm{Ru}$, and ${ }^{108} \mathrm{Pd}(n, \gamma){ }^{109} \mathrm{Pd}$ were dealt with in this study. From a comparison between the calculation (C) and the experiment (E) about ${ }^{102} \mathrm{Ru}(n, \gamma)^{103} \mathrm{Ru}$, the deviation (C/E-1) was significantly large. Then, it is strongly suspected that not MVP-BURN code but the neutron capture cross section of ${ }^{102} \mathrm{Ru}$ belonging to JENDL-4.0 used in this simulation have made the big difference as $(\mathrm{C} / \mathrm{E}-1)>20 \%$.
\end{abstract}

\section{Introduction}

The Après ORIENT research program [1] was initiated FY2011 aiming at creating stable, highly-valuable elements (defined as secondary nuclear rare metals; NRMs) by nuclear transmutation from fission products (FPs) separated from spent nuclear fuels. In our previous studies $[2,3]$ to analyse the efficiency of creation of NRMs and to estimate the radioactivity of created NRMs by $(n, \gamma)$ reaction followed by $\beta^{-}$decay of FPs in nuclear reactors, a simple burnup calculation had been performed by using ORLIBJ40 package [4], which is a combination of a burnup calculation code ORIGEN2 [5] and 1-group cross section library based on JENDL-4.0 [6]. As a result, a trend of efficiency of creation of NRM elements depending on the neutron capture cross section of target FP elements had become clear [2]. Then, as a comprehensive evaluation of created NRMs from view point of efficiency, radioactivity, and importance as resources, light platinum group elements, especially ruthenium $(\mathrm{Ru})$ and palladium (Pd), were specially selected as important NRMs, which should be much researched in the future works for realization of this advanced nuclear fuel cycle [3].

However, the calculation by using ORLIBJ40 was a simplified and ideal simulation as it was based on 1-group cross section and assumed homogeneous geometry. Thus, in order to realize a transmutation process,

a e-mail: terashima.a.aa@m.titech.ac.jp it is necessary to carry out a more detailed simulation to predict nuclear reactions in actual nuclear reactors more accurately. Then, a continuous-energy Monte Carlo burnup calculation code MVP-BURN [7] is one of the most appropriate tools because it has precise geometrical modeling and continuous-energy treatment.

In addition, a calculation code might have some errors even though the system and modeling are strict. Then, an evaluated nuclear data library used as a basic physical quantity might also have deviation from the actual value due to the lack of the experimental data. Thus, to validate a result of such simulation, an actual experiment to irradiate some elements with neutron in reactors is strongly requiered.

Therefore, in the present study, both an experiment of neutron activation analysis in TRIGA Mark I reactor at University of California, Irvine (UC Irvine) and the corresponding burnup calculation using MVP-BURN code were performed, which especially focused on light platinum group elements as analyzed targets.

\section{Methods}

\subsection{Irradiation experiment}

6 solutions containing different concentration of cation of $\mathrm{Ru}$ in dilute nitric acid were prepared from $\mathrm{Ru}$ atomic absorption standard solution (Sigma-Aldrich). In addition, 6 solutions containing different concentration of cation of 
$\mathrm{Pd}$ in dilute nitric acid were prepared from Pd standard for ICP (Sigma-Aldrich). Each $1 \mathrm{ml}$ of them was put in a short poly vial EP338-NAA (LA Container Inc.), and then it was also put in a long poly vial EP290-NAA (LA Container Inc.). Moreover, each the poly vial was put in a dedicated polyethylene container for TRIGA reactor.

For the position of the targets the rotary rack facility, so called "Lazy Susan", in TRIGA reactor at UC Irvine was selected. This rack is slowly rotated around just outside the reactor core but inside the graphite reflector surrounding the core. Then, thermal neutron flux is dominant at the position. In this irradiation experiment, all of the targets were irradiated at the same time in the "Lazy Susan" for 1 hour at $250 \mathrm{~kW}$.

After irradiation, gamma rays accompanying radioactive decay of ${ }^{103} \mathrm{Ru}\left(E_{\gamma} \mathcal{D} 497.1 \mathrm{keV}\right),{ }^{105} \mathrm{Ru}\left(E_{\gamma} \mathcal{D}\right.$ $724.3 \mathrm{keV})$, and ${ }^{109} \mathrm{Pd}\left(E_{\gamma} \mathcal{D} 88.0 \mathrm{keV}\right)$ were measured by a High-purity Germanium (HPGe) detector GC4018 (CANBERRA) and analysed by gamma analysis software Genie 2000 (CANBERRA). From the count per second of the representative gamma ray, the number of nucleus of each radioactive nuclide at the time of the end of the irradiation was calculated based on their decay history and the detector efficiency calibration.

\subsection{Burnup calculation}

In accordance with the above condition of the irradiation experiment by using TRIGA reactor at UC Irvine, a predictive simulation was performed by using MVPBURN code with a burnup calculation module added to 3dimensional Monte Carlo code based on continuous energy methods; MVP-III (beta-version) [8]. The 3-dimensional geometry in the simulation was constructed based on the actual reactor design [9-11]. It should be noted that, in order to handle the radioactive nuclides with short half-life, the burnup chain data of MVP-BURN (ChainJ40; u4cm6fp119bp14T_J40 ${ }^{\dagger}$ ) was modified around target nuclides, for example by adding creation and decay of ${ }^{109} \mathrm{Pd}$ to the burnup chain. It enabled to reproduce the experiment of neutron activation analysis for short-lived radioactive nuclides by MVP-BURN code.

Basically, MVPlib_nJ40 [12] based on JENDL-4.0 was used as a cross section library of all nuclides for MVP-BURN code. Moreover, for ${ }^{102} \mathrm{Ru},{ }^{104} \mathrm{Ru}$, and ${ }^{108} \mathrm{Pd}$ in the target region, not only MVPlib_nJ40 but also MVPlib_nB70 and MVPlib_nJ33 [13] were especially used which are based on ENDF/B-VII.0 [14] and JENDL-3.3 [15], respectively. From Table 1, for ${ }^{102} \mathrm{Ru},{ }^{104} \mathrm{Ru}$, and ${ }^{108} \mathrm{Pd}$, this combination of JENDL-4.0 and ENDF/B-VII.0 enables to compare among three latest evaluated libraries such as JENDL-4.0, ENDF/B-VII.1 [16], and JEFF-3.2 [17]. Then, MVPLIB_nJ33 was used in order to evaluate the neutron capture cross section of ${ }^{102} \mathrm{Ru}$, especially.

The number of history of neutron per batch was 160,000 , and the number of skip batch and effective batch were 100 and 1,000, respectively. The number of burnup step was 1 per hour. The number of sub-step was 120 per burn step.

In the above calculation condition, the number of nucleus of each the corresponding radioactive nuclide,

\footnotetext{
$\dagger$ "u4cm6fp119bp14T_J40" is one of the chain data file in ChainJ40, and can handle only 119 nuclides as fission products.
}

Table 1. Comparison of the evaluating laboratory and thermal neutron capture cross section of ${ }^{108} \mathrm{Pd},{ }^{102} \mathrm{Ru},{ }^{104} \mathrm{Ru}$, and ${ }^{96} \mathrm{Ru}$ in three latest evaluated nuclear data libraries and JENDL-3.3.

\begin{tabular}{|c|c|c|c|}
\hline & \multicolumn{3}{|c|}{ Laboratory having evaluated the nuclear data } \\
\hline & \multicolumn{3}{|c|}{ Thermal neutron capture cross section [b] } \\
\hline & $\begin{array}{c}\text { JENDL-4.0 } \\
\text { (JENDL-3.3) }\end{array}$ & ENDF/B-VII.1 & JEFF-3.2 \\
\hline \multirow{3}{*}{${ }^{108} \mathrm{Pd}$} & JNDC & LANL, BNL & LANL, BNL \\
\hline & $\begin{array}{c}8.05 \\
(8.507)\end{array}$ & $\begin{array}{c}8.48 \\
\text { same to }\end{array}$ & $\begin{array}{c}8.48 \\
\text { same to }\end{array}$ \\
\hline & & ENDF/B-VII.0 & ENDF/B-VII.0 \\
\hline \multirow{3}{*}{${ }^{102} \mathrm{Ru}$} & JNDC & CNDC & CNDC \\
\hline & $\begin{array}{c}1.48 \\
(1.230)\end{array}$ & $\begin{array}{c}1.27 \\
\text { same to }\end{array}$ & $\begin{array}{c}1.27 \\
\text { same to }\end{array}$ \\
\hline & & ENDF/B-VII.0 & ENDF/B-VII.0 \\
\hline \multirow{4}{*}{${ }^{104} \mathrm{Ru}$} & JNDC & CNDC & CNDC \\
\hline & 0.469 & 0.472 & 0.472 \\
\hline & $(0.328)$ & same to & same to \\
\hline & & ENDF/B-VII.0 & ENDF/B-VII.0 \\
\hline \multirow[b]{2}{*}{${ }^{96} \mathrm{Ru}$} & JNDC & JNDC & NEA \\
\hline & $\begin{array}{c}0.271 \\
(0.290)\end{array}$ & $\begin{array}{c}0.290 \\
\text { same to } \\
\text { JENDL-3.3 }\end{array}$ & 0.249 \\
\hline
\end{tabular}

namely ${ }^{103} \mathrm{Ru},{ }^{105} \mathrm{Ru}$, and ${ }^{109} \mathrm{Pd}$, at the time of the end of the irradiation was calculated.

\subsection{Comparison between burnup calculation and irradiation experiment about ${ }^{108} \mathrm{Pd}(n, \gamma)^{109} \mathrm{Pd}$}

Because concentration of cations of $\mathrm{Pd}$ in the standard for ICP was good precision, the result from burnup calculation of $\mathrm{Pd}$ was compared to the result from irradiation experiment of $\mathrm{Pd}$ as it is.

\subsection{Comparison between burnup calculation and irradiation experiment about ${ }^{102} \mathrm{Ru}(n, \gamma)^{103} \mathrm{Ru}$}

Because concentration of cation of $\mathrm{Ru}$ in the atomic absorption standard solution was not good precision, it is needed to correct the deviation of concentration of $\mathrm{Ru}$ before comparison between calculation and experiment. As one method of the correction, the relative evaluation can be applied, which the reaction rate of an isotope is normalized by that of another isotope.

Actually, in one of past measurements of neutron capture cross section of ${ }^{102} \mathrm{Ru}$, the cross section of ${ }^{96} \mathrm{Ru}$ had been used as normalization factor [18]. However, it is considered that using ${ }^{96} \mathrm{Ru}$ as normalization factor is not good because the cross section has comparatively big difference as $10 \%$ among three latest evaluated libraries (cf. Table 1). Thus, according to the selection of evaluated library as standard for the relative evaluation, the result could be change drastically.

On the other hand, neutron capture cross section of ${ }^{104} \mathrm{Ru}$ has comparatively small difference as $1 \%$ among three latest evaluated libraries (cf. Table 1). Thus, even if another evaluated library as standard for the relative evaluation is used, only a little change from the default result could be caused. Therefore, in the present study, ${ }^{104} \mathrm{Ru}(n, \gamma){ }^{105} \mathrm{Ru}$ reaction was used as a normalization factor for $\left.{ }^{102} \mathrm{Ru}(n, \gamma)\right)^{103} \mathrm{Ru}$ reactions to correct the difference of concentration of $\mathrm{Ru}$ in the sample solution 


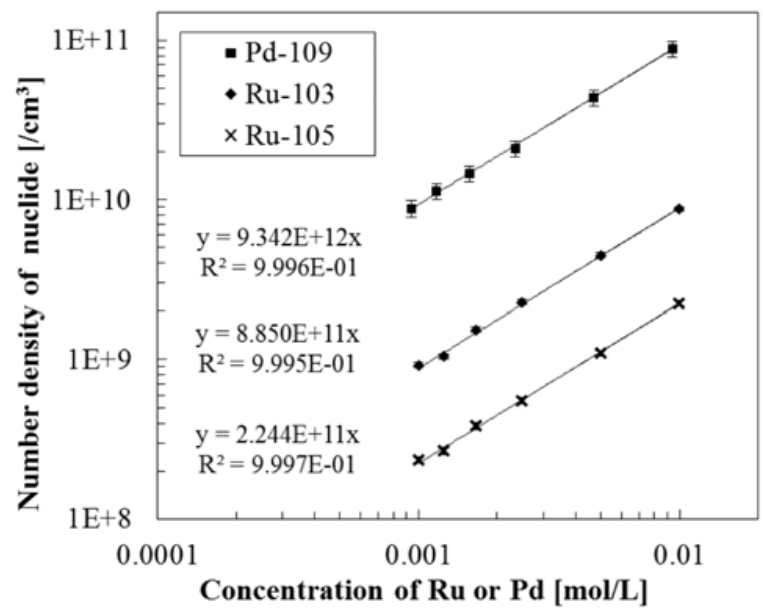

Figure 1. Number density of created radioactive nuclides at $t_{i}$ vs. concentration of cation of $\mathrm{Ru}$ or Pd in each sample solution.

between in the calculation condition and in the actual condition.

\section{Results and discussion}

\subsection{Experimental results}

From the measurement of gamma rays accompanying radioactive decay of ${ }^{103} \mathrm{Ru},{ }^{105} \mathrm{Ru}$, and ${ }^{109} \mathrm{Pd}$, the number density of each of them at the time of the end of the irradiation was calculated by Eq. (1);

$$
\begin{aligned}
N\left(t_{i}\right) \mathcal{D} & \frac{1}{\lambda} \times A\left(t_{i}\right) \\
\mathcal{D} & \frac{1}{\lambda} \times \frac{\operatorname{CPS}\left(t_{d}\right)}{1 \times \varepsilon} \\
& \times \exp \left[\lambda\left(t_{d}-t_{i}\right)\right] \times \frac{\lambda\left(t_{c}-t_{d}\right)}{1-\exp \left[-\lambda\left(t_{c}-t_{d}\right)\right]}
\end{aligned}
$$

where, $N\left(t_{i}\right)\left[/ \mathrm{cm}^{3}\right]$ is the number density of nucleus of created radioactive nuclide at the time of the end of the irradiation $\left(t_{i}[\mathrm{~s}]\right), A\left(t_{i}\right)[\mathrm{Bq}]$ is the radioactivity of the nuclide at $t_{i}, t_{d}$ [s] is the time of the beginning of the counting the gamma ray, $t_{c}[\mathrm{~s}]$ is the time of the end of the counting, $I[\%]$ is the intensity of the gamma ray, and $\varepsilon[\%]$ is the efficiency of the detector depending on the position and shape of the sample and the energy of the gamma ray. It should be noted, in this paper, following four uncertainties were considered; from experiment, count per second $C P S$ and detector efficiency $\varepsilon$; from nuclear data, intensity $I$ and decay constant $\lambda$.

Figure 1 shows the result of number density of created radioactive nuclides at $t_{i}$ vs. concentration of cation of $\mathrm{Ru}$ or $\mathrm{Pd}$ in each sample solution. It turns out, for all of the three nuclides, all of the 6 measurements on each concentration are considered to be a success because each $\mathrm{R}^{2}$ value is very close to 1 .

\subsection{Results of $\mathrm{C} / \mathrm{E}$ evaluation for ${ }^{109} \mathrm{Pd}$}

Figure 2 shows the result of the absolute evaluation of $\mathrm{C} / \mathrm{E}$ (Calculated value; $\mathrm{C}$, Experimental value; $\mathrm{E}$ ) of the number density of created ${ }^{109} \mathrm{Pd}$. In this figure, the total uncertainty considering both uncertainties of $\mathrm{C}$ and $\mathrm{E}$ is shown as the error bar. As the statistic error of simulation is

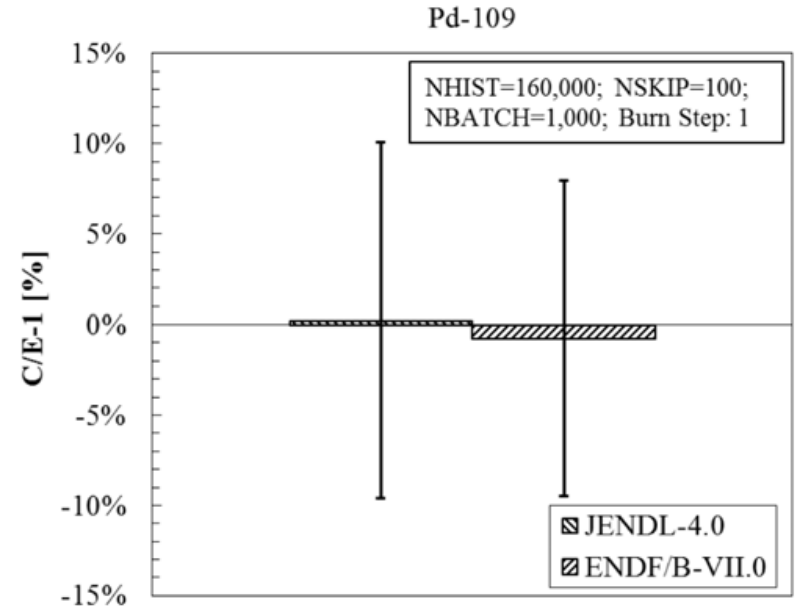

Figure 2. Comparison of $\mathrm{C} / \mathrm{E}$ values of number density of created ${ }^{109} \mathrm{Pd}$ in the case using JENDL-4.0 and ENDF/B-VII.0.

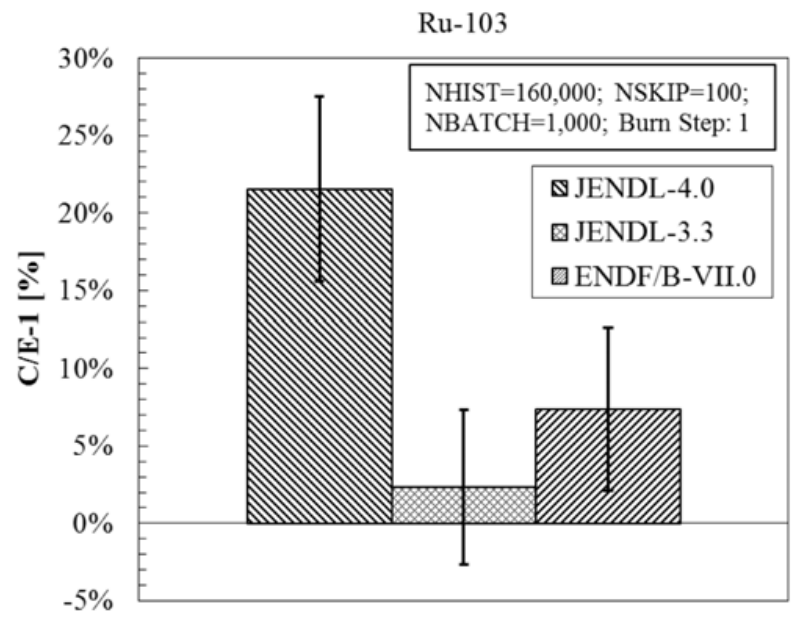

Figure 3. Comparison of $\mathrm{C} / \mathrm{E}$ values of number density of created ${ }^{103} \mathrm{Ru}$ in the case using JENDL-4.0, -3.3 and ENDF/B-VII.0.

only $1 \%$, the main cause of the total error is the uncertainty of the intensity of the measured gamma ray; I D 3.6(4)\% $\left(E_{\gamma} \mathcal{D} 88.0 \mathrm{keV}\right)$ [19]. It turns out that both libraries for ${ }^{108} \mathrm{Pd}(n, \gamma){ }^{109} \mathrm{Pd}$ obtained good results of $(\mathrm{C} / \mathrm{E}-1)$ near zero. In the case of ${ }^{108} \mathrm{Pd}$, it would be considered that the deference of thermal cross section in both libraries shown in Table 1 was cancelled by that of some large resonances at lower than $100 \mathrm{eV}$. Therefore, MVP-BURN code with modified burnup chain data could be considered to enable us to simulate the neutron activation analysis if the sample with high accuracy and a reasonable evaluated library are used.

\subsection{Results of $C / E$ evaluation for ${ }^{103} R u$}

Figure 3 shows the result of the relative evaluation of $\mathrm{C} / \mathrm{E}$ of the number density of created ${ }^{103} \mathrm{Ru}$. In this figure, the total uncertainty is shown as the error bar similar to that in Fig. 2. It turns out that these values of $(\mathrm{C} / \mathrm{E}-1)$ in the case of JENDL-4.0 and ENDF/B-VII.0 were over than $20 \%$ and $7 \%$, respectively. Moreover, the point of C/E $\mathcal{D} 1$ did not enter in the range of the total error bar not only in the case of JENDL-4.0 but also in that of ENDF/BVII.0. Thus, this fact suggests the possibility that both 


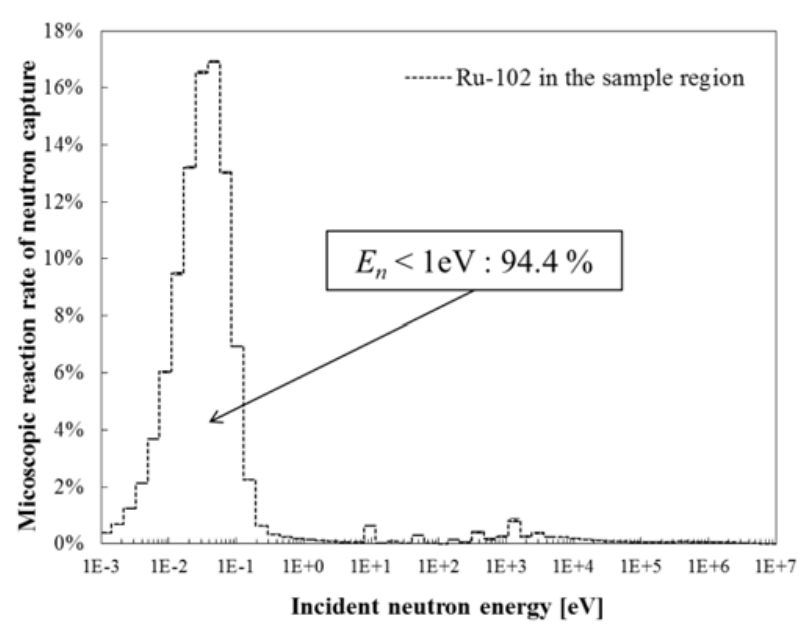

Figure 4. Simulated microscopic reaction rate of neutron capture of ${ }^{102} \mathrm{Ru}$ in the sample region for irradiation.

libraries overestimate the thermal neutron capture cross section because thermal neutron flux is dominant in the sample region for irradiation (cf. Fig. 4).

As all the results of $\mathrm{C} / \mathrm{E}$ values of ${ }^{103} \mathrm{Ru}$ from three libraries, the thermal neutron capture cross section of ${ }^{102} \mathrm{Ru}$ have been estimated as 1.21(3) b. Then, the range of the total uncertainty of this estimated value includes the latest measured value 1.241(1) b by F. F. Arboccò, et al. in 2014 [20]. However, it should be noted, it has a possibility the estimated value may change if the data of ${ }^{104} \mathrm{Ru}$ from another library is used for the normalization in this method. Thus, such evaluation would be performed in the future work to improve this verification of the thermal neutron capture cross section of ${ }^{102} \mathrm{Ru}$.

\section{Conclusions}

In the present study, a burnup calculation by MVP-BURN code with MVP-III (beta-version) was adopted to analysis of a neutron activation experiment at UCI-TRIGA reactor. As a absolute $\mathrm{C} / \mathrm{E}$ estimation of the amount of created ${ }^{109} \mathrm{Pd}$ by capture reaction of ${ }^{108} \mathrm{Pd}$, it turned out that MVPBURN code with modified burnup chain data would enable us to simulate the neutron activation analysis for a shortlived radioactive isotope. In addition, as a relative $\mathrm{C} / \mathrm{E}$ estimation of the amount of created ${ }^{103} \mathrm{Ru}$ by capture reaction of ${ }^{102} \mathrm{Ru}$, it pointed out the possibility that the data of thermal neutron capture cross section of ${ }^{102} \mathrm{Ru}$ in JENDL-4.0 and ENDFB-VII.0 could be overestimated more than $20 \%$ and $7 \%$, respectively. Then, based on 3 values of C/E from JENDL-4.0, JENDL-3.3, and ENDF/BVII.0, the thermal neutron capture cross section of ${ }^{102} \mathrm{Ru}$ was estimated as $1.21 \pm 0.03 \mathrm{~b}$. Thus, this estimation by combination of experiment and simulation supported the latest measured value $1.241 \mathrm{~b}$. In the future work, the simulation using the data of ${ }^{104} \mathrm{Ru}$ from another library would be performed in order to improve this verification method much more.

The authors would like to sincerely thank Dr. Yasunobu Nagaya (Japan Atomic Energy Agency) for his many more technical advices about calculation by MVP code. This work was a part of the research supported by JSPS KAKENHI Grant Number 15J12066 (Grant-in-Aid for JSPS Fellows) and Academy for Co-creative Education of Environment and Energy Science (ACEEES), Tokyo Institute of Technology, Japan.

\section{References}

[1] M. Ozawa, C.Y. Han, T. Kaneshiki, M. Nilsson, Proceedings of International Nuclear Fuel Cycle Conference (GLOBAL 2013), Salt Lake City, Utah, USA, Sep.29-Oct.3, 2013, pp. 1499-1503 (2013)

[2] A. Terashima, M. Ozawa, Nucl. Sci. Tech. 26, S010311 (2015)

[3] A. Terashima, M. Ozawa, Prog. Nucl. Energy 93, 177-185 (2016)

[4] K. Okumura, K. Sugino, K. Kojima, et al., JAEAData/Code 2012-032 (Japan Atomic Energy Agency, 2013)

[5] A.G. Croff, Nucl. Technol. 62, 335-352 (1983)

[6] K. Shibata, O. Iwamoto, T. Nakagawa, et al., J. Nucl. Sci. Technol. 48, 1-30 (2011)

[7] K. Okumura, T. Mori, M. Nakagawa, K. Kaneko, J. Nucl. Sci. Technol. 37, 128-138 (2000)

[8] Y. Nagaya, K. Okumura, T. Mori, M. Nakagawa, JAERI 1348 (Japan Atomic Energy Research Institute, 2005)

[9] J.R. Humphries, GA 9389 (General Atomics, 1969)

[10] G.E. Miller, Safety Analysis Report Revision 1 (University of California, Irvine, 1999)

[11] J. Crozier, GA 911196 (General Atomics, 2010)

[12] K. Okumura, Y. Nagaya, JAEA-Data/Code 2011010, (Japan Atomic Energy Agency, 2011)

[13] T. Mori, Y. Nagaya, K. Okumura, K. Kaneko, JAERI-Data/Code 2004-011 (Japan Atomic Energy Research Institute, 2004)

[14] M.B. Chadwick, P. Obložinský, M. Herman, et al., Nucl. Data Sheets 107, 2931-3060 (2006)

[15] K. Shibata, T. Kawano, T. Nakagawa, et al., J. Nucl. Sci. Technol. 32, 1125-1136 (2002)

[16] M.B. Chadwick, M. Herman, P. Obložinský, et al., Nucl. Data Sheets 112, 2887-2996 (2011)

[17] JEFF-3.2 evaluated data library - Neutron data, OECD-NEA, https : //www.oecd-nea.org/dbforms / data/eva/evatapes/jeff_32/ (Last access: Oct. 26, 2016)

[18] H. Ishikawa, J. Nucl. Sci. Technol. 6, 587-590 (1969)

[19] Live Chart of Nuclides, IAEA, https://www-nds. iaea.org/relnsd/vcharthtml/VChartHTML . html (Last access: Oct. 26, 2016)

[20] F.F. Arboccò, P. Vermaercke, K. Smits, et al., J. Radioanal. Nucl. Chem. 302, 655-672 (2014) 